\title{
Baryonic B Decays
}

\section{Jing-Ge Shiu*}

National Taiwan University, Taiwan

E-mail: phys jg@hep1.phys.ntu . edu . tw

We present a summary of the recent studies on the baryonic B decays performed by the BaBar and Belle collaborations. Several new decays have been observed in experiments. The hierarchy in branching fractions of baryonic B decays in both charmed and charmless decays are established. The near-threshold-enhancement in the baryon-antibaryon mass distributions for three- and fourbody decay is also checked. In the charmless decays, differences are seen in the $B \rightarrow p \bar{p} h$ and $B \rightarrow \Lambda \bar{\Lambda} h$ decays, which indicate different underlying dynamics may exist. A preliminary result of first semileptonic B decay is also presented.

Flavor Physics and CP Violation - FPCP 2010

May 25-29, 2010

Turin, Italy

${ }^{*}$ Speaker. 


\section{Introduction}

After several years of B factories running, the B factories, BaBar and Belle, have collected a large data sample of $B \bar{B}$ pairs at the $\Upsilon(4 S)$ resonance produced in asymmetric $e^{+} e^{-}$collisions. It provides an opportunity to study the properties of baryonic B meson decays, which are less understood than $\mathrm{B}$ decays into mesons.

An intriguing feature of baryonic B decays has been established experimentally: the hierarchy in branching fractions of baryonic B decays. The observed branching fraction of two-body decays is about one order of magnitude less than the corresponding three-body decays. Several theoretical approaches have tried to calculate the baryonic B decays. The most satisfying one is the qualitative one proposed by Hou and Soni in 2001 [1]. The baryonic B decays are favored when the baryon and antibaryon are close together in phase space. This is also supported by the experimental observation, in three- or four-body decays, of enhancement in the partial branching fraction near the baryon-antibaryon mass threshold.

The profoundity of baryonic decays is an unique feature of B meson, and a searching ground for possible exotic states and new physics. In this report, we summarized the recent results in charmed and charmless baryonic B decay studies. The detail of each analysis is beyond the scope of this report.

\section{Charmed baryonic decays}

\section{1 $\bar{B}^{0} \rightarrow \Lambda_{c}^{+} \bar{p}$ and $B^{-} \rightarrow \Lambda_{c}^{+} \bar{p} \pi^{-}$}

BaBar collaboration has pursued an analysis of $\bar{B}^{0} \rightarrow \Lambda_{c}^{+} \bar{p}$ and $B^{-} \rightarrow \Lambda_{c}^{+} \bar{p} \pi^{-}$decays [2], using a data sample of 383 million $B \bar{B}$ events [3]. The measured branching fractions of these two modes, together with previous measurements done by Belle collaboration with less statistics $[4,5]$, are listed in Table 1. Both experiments use the same value for $\mathscr{B}\left(\Lambda_{c}^{+} \rightarrow p K^{-} \pi^{+}\right)$. If combining the statistical and systematic uncertainties only, the $\mathscr{B}\left(\bar{B}^{0} \rightarrow \Lambda_{c}^{+} \bar{p}\right)$ measurements by the two collaborations agree with each other. On the other hand, the newly measured branching fraction for the three-body mode is significant larger than the previous one done by Belle collaboration.

\begin{tabular}{|l|c|c|}
\hline & $\mathscr{B}\left(\bar{B}^{0} \rightarrow \Lambda_{c}^{+} \bar{p}\right)\left(x 10^{-5}\right)$ & $\mathscr{B}\left(B^{-} \rightarrow \Lambda_{c}^{+} \bar{p} \pi^{-}\right)\left(x 10^{-4}\right)$ \\
\hline BaBar & $1.89 \pm 0.21 \pm 0.06 \pm 0.49$ & $3.38 \pm 0.12 \pm 0.12 \pm 0.88$ \\
\hline Belle & $2.19_{-0.49}^{+0.56} \pm 0.32 \pm 0.57$ & $2.01 \pm 0.15 \pm 0.20 \pm 0.52$ \\
\hline
\end{tabular}

Table 1: The branching fractions of $\bar{B}^{0} \rightarrow \Lambda_{c}^{+} \bar{p}$ and $B^{-} \rightarrow \Lambda_{c}^{+} \bar{p} \pi^{-}$measured by BaBar and Belle collaborations. The uncertainties are statistical, systematic, and the uncertainty in $\mathscr{B}\left(\Lambda_{c}^{+} \rightarrow p K^{-} \pi^{+}\right)$respectively.

The ratio of the branching fractions is

$$
\frac{\mathscr{B}\left(B^{-} \rightarrow \Lambda_{c}^{+} \bar{p} \pi^{-}\right)}{\mathscr{B}\left(\bar{B}^{0} \rightarrow \Lambda_{c}^{+} \bar{p}\right)}=15.4 \pm 1.8 \pm 0.3,
$$

where most of the systematic uncertainties, including the $\mathscr{B}\left(\Lambda_{c}^{+} \rightarrow p K^{-} \pi^{+}\right)$, cancel [6]. This is consistent with the hierarchy in branching fractions observed in other baryonic B decays. An enhancement of the decay rate near the $\Lambda_{c}^{+} \bar{p}$ mass threshold is also observed. 


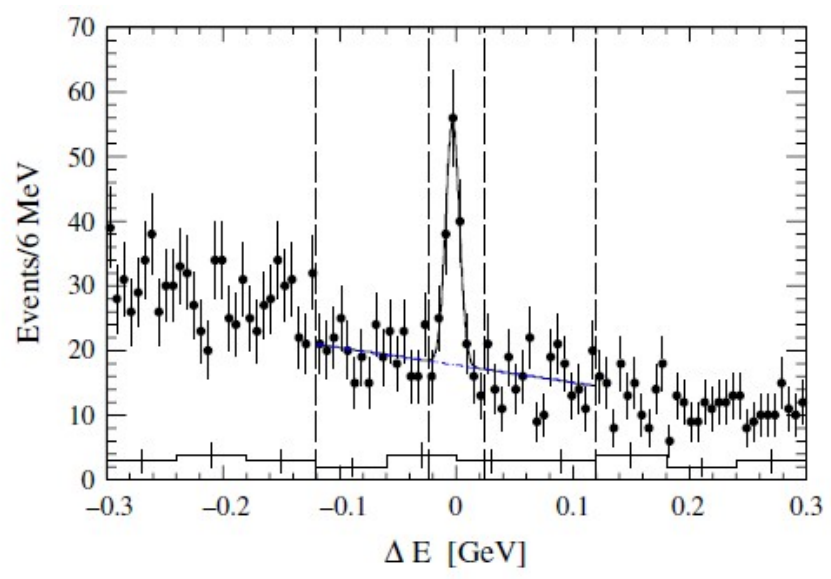

Figure 1: Fitted $\Delta E$ distribution with data points for the $\bar{B}^{0} \rightarrow \Lambda_{c}^{+} \bar{p} K^{-} \pi^{+}$study. The continuum background, described by off-resonance data, is overlaid (histogram). The vertical dashed lines indicate the signal and sideband regions.

In addition, they have also measured the fractions of $B^{-} \rightarrow \Lambda_{c}^{+} \bar{p} \pi^{-}$decays that proceed through a quasi two-body decays $\Sigma_{c} \rightarrow \Lambda_{c}^{+} \pi^{-}$:

$$
\begin{aligned}
& \frac{\mathscr{B}\left(B^{-} \rightarrow \Sigma_{c}(2455)^{0} \bar{p}\right)}{\mathscr{B}\left(B^{-} \rightarrow \Lambda_{c}^{+} \bar{p} \pi^{-}\right)}=(12.3 \pm 1.2 \pm 0.8) \times 10^{-2}, \\
& \frac{\mathscr{B}\left(B^{-} \rightarrow \Sigma_{c}(2800)^{0} \bar{p}\right)}{\mathscr{B}\left(B^{-} \rightarrow \Lambda_{c}^{+} \bar{p} \pi^{-}\right)}=(11.7 \pm 2.3 \pm 2.4) \times 10^{-2}, \\
& \frac{\mathscr{B}\left(B^{-} \rightarrow \Sigma_{c}(2520)^{0} \bar{p}\right)}{\mathscr{B}\left(B^{-} \rightarrow \Lambda_{c}^{+} \bar{p} \pi^{-}\right)}<0.9 \times 10^{-2} \text { at } 90 \% \text { C.L. }
\end{aligned}
$$

This is the first observation of $B^{-} \rightarrow \Sigma_{c}(2800)^{0} \bar{p}$ decays. The spin of the $\Sigma_{c}(2455)^{0}$ is also examined to be $J=1 / 2$, which is consistent with the expectation for the lowest $\Sigma_{c}$ baryon state.

1.2 $\bar{B}^{0} \rightarrow \Lambda_{c}^{+} \bar{p} K^{-} \pi^{+}$

An observation of the Cabibbo-suppressed decay $\bar{B}^{0} \rightarrow \Lambda_{c}^{+} \bar{p} K^{-} \pi^{+}$is reported by BaBar collaboration (Figure 1), based on their full data set of 467 million $B \bar{B}$ pairs [7]. The measured branching fraction is

$$
\mathscr{B}\left(\bar{B}^{0} \rightarrow \Lambda_{c}^{+} \bar{p} K^{-} \pi^{+}\right)=(4.33 \pm 0.82 \pm 0.33 \pm 1.13) \times 10^{-5}
$$

where the uncertainties are statistical, systematic, and the uncertainty in $\mathscr{B}\left(\Lambda_{c}^{+} \rightarrow p K^{-} \pi^{+}\right)$. Additionally, by checking the mass distributions of $\Lambda_{c}^{+} \pi^{+}$and $K^{-} \pi^{+}$pairs, they also find evidence for the resonant subchannel $\bar{B}^{0} \rightarrow \Sigma_{c}(2455)^{++} \bar{p} K^{-}$and set an upper limit for $\bar{B}^{0} \rightarrow \Lambda_{c}^{+} \bar{p} K^{* 0}$.

$$
\begin{gathered}
\mathscr{B}\left(\bar{B}^{0} \rightarrow \Sigma_{c}(2455)^{++} \bar{p} K^{-}\right)=(1.11 \pm 0.30 \pm 0.09 \pm 0.29) \times 10^{-5}, \\
\mathscr{B}\left(\bar{B}^{0} \rightarrow \Lambda_{c}^{+} \bar{p} K^{* 0}\right)<2.42 \times 10^{-5} \text { at } 90 \% \text { C.L. }
\end{gathered}
$$


The measured branching fractions of the two Cabibbo-suppressed decays can be compared with those of the Cabibbo-favored decays $\bar{B}^{0} \rightarrow \Lambda_{c}^{+} \bar{p} \pi^{-} \pi^{+}$and $\bar{B}^{0} \rightarrow \Sigma_{c}(2455)^{++} \bar{p} \pi^{+}$, which have been observed in other experiments $[8,9]$. For the three-body decay, the ratio of the Cabibbosuppressed to favored decays is $0.048 \pm 0.016$, compatible with the $\left|V_{u s} / V_{u d}\right|^{2}=0.0536 \pm 0.0020$ [10], where $V_{u s}$ and $V_{u d}$ are Cabibbo-Kobayashi-Maskawa matrix elements. However, the ratio for the four-body decays is $0.038 \pm 0.009$, which is smaller than $\left|V_{u s} / V_{u d}\right|^{2}$. This may indicate some additional decay amplitudes in the Cabibbo-favored decays.

\subsection{Semileptonic decays into $\Lambda_{c}^{+}$}

A preliminary study on B semileptonic decays into $\Lambda_{c}^{+}$is presented by BaBar collaboration, base on 460 million $B \bar{B}$ pairs [13]. If the charmed baryonic production is same as the mesonics production $B \rightarrow D X$, dominated by emission of an external $W$ boson, then one would expect the ratio of semileptonic events to hardronic events to be similar for the baryonic and mesonic processes:

$$
\frac{\mathscr{B}\left(\bar{B} \rightarrow \Lambda_{c}^{+} X \ell^{-} \bar{v}_{\ell}\right)}{\mathscr{B}\left(\bar{B} \rightarrow \Lambda_{c}^{+} / \bar{\Lambda}_{c}^{-} X\right)} \sim \frac{\mathscr{B}\left(\bar{B} \rightarrow D X \ell^{-} \bar{v}_{\ell}\right)}{\mathscr{B}(\bar{B} \rightarrow D / \bar{D} X)}
$$

The Feynman diagrams of B decays with internal and external $W$ emissions are shown in Figure 2.

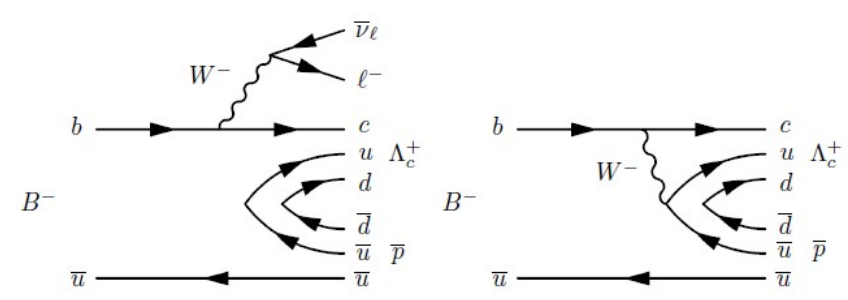

Figure 2: B decays with an external (left) and internal (right) $W$ emission.

For the mesonic process, the ratio is about $10 \%$ [14]. The preliminary results of this study are

$$
\begin{aligned}
& \frac{\mathscr{B}\left(\bar{B} \rightarrow \Lambda_{c}^{+} X e^{-} \bar{v}_{e}\right)}{\mathscr{B}\left(\bar{B} \rightarrow \Lambda_{c}^{+} / \bar{\Lambda}_{c}^{-} X\right)}=(3.9 \pm 1.0 \pm 1.1) \%, \\
& \frac{\mathscr{B}\left(\bar{B} \rightarrow \Lambda_{c}^{+} X \mu^{-} \bar{v}_{\mu}\right)}{\mathscr{B}\left(\bar{B} \rightarrow \Lambda_{c}^{+} / \bar{\Lambda}_{c}^{-} X\right)}=(1.57 \pm 1.7 \pm 1.7) \%,
\end{aligned}
$$

and the combined result is

$$
\frac{\mathscr{B}\left(\bar{B} \rightarrow \Lambda_{c}^{+} X \ell^{-} \bar{v}_{\ell}\right)}{\mathscr{B}\left(\bar{B} \rightarrow \Lambda_{c}^{+} / \bar{\Lambda}_{c}^{-} X\right)}=(3.2 \pm 0.9 \pm 0.9) \% .
$$

This is the first evidence for semileptonic B decays into $\Lambda_{c}^{+}$. The observed relative branching fraction is smaller than that for the mesonic decays which have a D meson in the final state. This may indicate significant contribution from internal $W$ emission in the baryonic producion. 


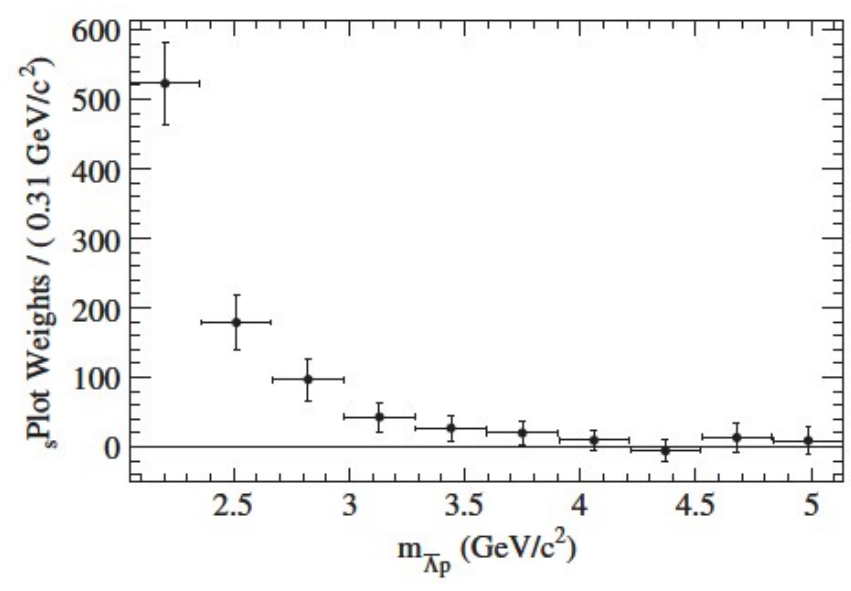

Figure 3: The $m(p \bar{\Lambda})$ event rate distribution in the $B^{0} \rightarrow p \bar{\Lambda} \pi^{-}$study.

\section{Charmless baryonic decays}

Unlike the charmed baryonic $\mathrm{B}$ decays proceeding through $b \rightarrow c$ tree diagrams, the charmless baryonic B decays presumably proceed via $b \rightarrow s$ penguin or/and $b \rightarrow u$ tree diagrams. The $b \rightarrow s$ penguin loop plays an important role in the charmless B meson decays [11]. It may have unexpectedly large $\mathrm{CP}$ violation or other new physics beyond the standard model.

\section{$2.1 B^{0} \rightarrow p \bar{\Lambda} \pi^{-}$}

The BaBar collaboration has measured the $\mathscr{B}\left(B^{0} \rightarrow p \bar{\Lambda} \pi^{-}\right)=(3.07 \pm 0.31 \pm 0.23) \times 10^{-6}$, based on their full data sample of 467 million $B \bar{B}$ pairs [12]. This result agrees with a previous measurement by Belle collaboration [15]. An enhancement in the differential spectrum near the invariant mass of the baryon-antibaryon pair $p \bar{\Lambda}$ is also confirmed (Figure 3 ).

They have also studied the $\bar{\Lambda}$ polarization as a function of $\bar{\Lambda}$ energy in the $B^{0}$ rest frame $\left(E_{\bar{\Lambda}}^{*}\right)$. The result is compatible with the theoretical prediction of full longitudinal right-handed polarization at large $E_{\bar{\Lambda}}^{*}$ (Figure 4).

\section{$2.2 B \rightarrow \Lambda \bar{\Lambda} h$}

Belle collaboration have examined the charmless decays $B \rightarrow \Lambda \bar{\Lambda} h$, where $h$ stands for $\pi^{+}$, $K^{+}, K^{0}, K^{*+}$, or $K^{* 0}$, based on a data sample of 657 million $B \bar{B}$ pairs [16]. For the decays having a $K / K^{*}$ in the final states, one s quark is produced through $b \rightarrow s$ penguin loop process and another two $s$ quarks from either a $s d-\bar{d} \bar{s}$ pair or a $s \bar{s}$ pair popping up from vacuum (Figure 5).

The $b \rightarrow s$ penguin loop process could be sensitive to new physics beyond the standard model due to additional contributions from yet-undiscovered heavy virtual particles. Recent studies in $B \rightarrow p \bar{p} h$ [17] and $B \rightarrow p \bar{\Lambda} h$ [15] gave intriguing results. It seems no single theoretic model can well interpret all the observations established in experiments. The proton polar angular distribution in the baryon-antibaryon helicity frame disagree with the predictions for short distance $b \rightarrow s$ weak decays [18]. The predicted branching fraction of $B \rightarrow p \bar{p} K^{* 0}$ from pole model is about a factor of 20 smaller then the measurement [19]. Therefore it is interesting to study the corresponding 


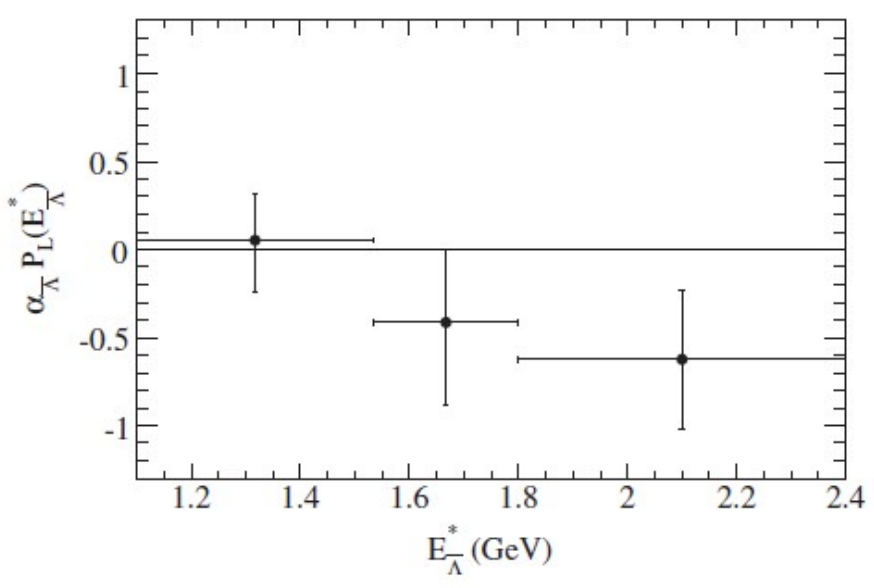

Figure 4: The product of $\bar{\Lambda}$ logitudinal polarization and $\alpha_{\bar{\Lambda}}$ as a function of $E_{\bar{\Lambda}}^{*}$. Horizontal bars represent bin ranges. The $\alpha_{\bar{\Lambda}}$ is the $\bar{\Lambda}$ decay-asymmetry parameter [10].
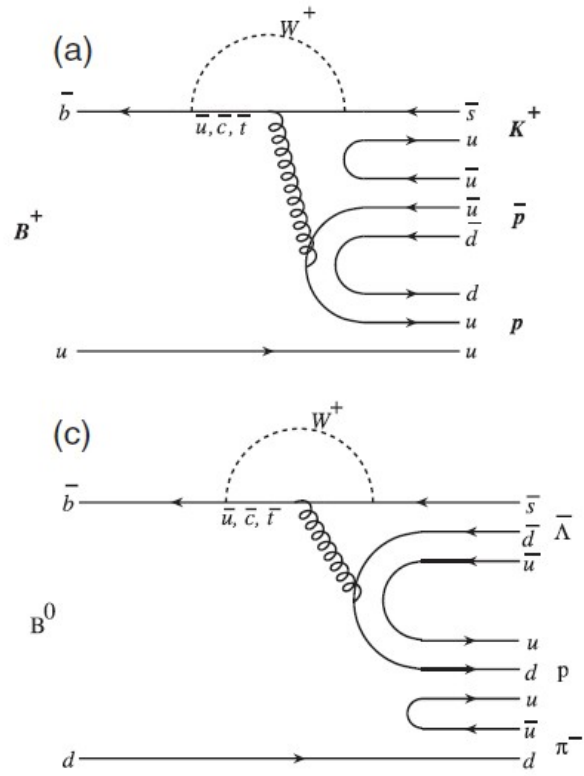
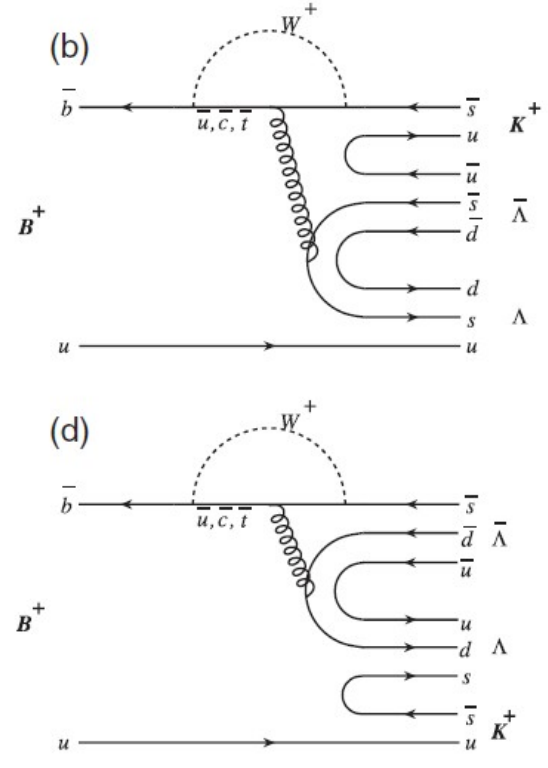

Figure 5: Comparisons of possible decay diagrams between $B^{+} \rightarrow p \bar{p} K^{+} / B^{0} \rightarrow p \bar{\Lambda} \pi^{-}$and $B^{+} \rightarrow \Lambda \bar{\Lambda} K^{+}$.

$B \rightarrow \Lambda \bar{\Lambda} h$ states, the counterparts with protons replaced by $\Lambda$ (Figure 5), in order to understand the dominant underlying physics.

The results of this study are summarized in Table 2, together with corresponding $B \rightarrow p \bar{p} h$ results from previous studies $[17,20]$. The enhancement near the invariant mass of $\Lambda \bar{\Lambda}$ pair is confirmed or with strong hint in all modes. Two modes, $B^{0} \rightarrow \Lambda \bar{\Lambda} K^{0}$ and $B^{0} \rightarrow \Lambda \bar{\Lambda} K^{* 0}$, are observed the first time. The branching fraction of $B^{+} \rightarrow \Lambda \bar{\Lambda} K^{+}$is updated with better accuracy, and a measurement or $90 \%$ confidence level upper limit in the threshold-mass-enhanced region $\left(M_{\Lambda \bar{\Lambda}}<2.85 \mathrm{GeV} / \mathrm{c}^{2}\right)$ for the other two modes are reported. 


\begin{tabular}{|c|c|c|c|c|}
\hline \multicolumn{2}{|c|}{$\mathscr{B}(B \rightarrow p \bar{p} h)\left(10^{-6}\right)$} & \multicolumn{2}{|c|}{$\mathscr{B}(B \rightarrow \Lambda \bar{\Lambda} h)\left(10^{-6}\right)$} & significances $(\sigma)$ \\
\hline$B^{0} \rightarrow p \bar{p} K^{0}$ & $2.51_{-0.29}^{+0.35} \pm 0.21$ & $B^{0} \rightarrow \Lambda \bar{\Lambda} K^{0}$ & $4.76_{-0.68}^{+0.84} \pm 0.61$ & 12.5 \\
\hline$B^{0} \rightarrow p \bar{p} K^{* 0}$ & $1.18_{-0.25}^{+0.29} \pm 0.11$ & $B^{0} \rightarrow \Lambda \bar{\Lambda} K^{* 0}$ & $2.46_{-0.72}^{+0.89} \pm 0.34$ & 9.0 \\
\hline$B^{+} \rightarrow p \bar{p} K^{+}$ & $5.54_{-0.25}^{+0.27} \pm 0.36$ & $B^{+} \rightarrow \Lambda \bar{\Lambda} K^{+}$ & $3.38_{-0.36}^{+0.41} \pm 0.41$ & 16.5 \\
\hline$B^{+} \rightarrow p \bar{p} K^{*+}$ & $3.38_{-0.60}^{+0.73} \pm 0.39$ & $B^{+} \rightarrow \Lambda \bar{\Lambda} K^{*+}$ & $\begin{array}{l}2.19_{-0.88}^{+1.13} \pm 0.33 \\
(<4.98 \text { at } 90 \% \text { C.L. })\end{array}$ & 3.7 \\
\hline$B^{+} \rightarrow p \bar{p} \pi^{+}$ & $1.60_{-0.19}^{+0.22} \pm 0.12$ & $B^{+} \rightarrow \Lambda \bar{\Lambda} \pi^{+}$ & $<0.94$ at $90 \%$ C.L. & 2.5 \\
\hline
\end{tabular}

Table 2: Summary of all $B \rightarrow p \bar{p} h$ (left) and $B \rightarrow \Lambda \bar{\Lambda} h$ (right) results. The $B^{+} \rightarrow \Lambda \bar{\Lambda} K^{*+}$ and $B^{+} \rightarrow \Lambda \bar{\Lambda} \pi^{+}$ results are for the threshold-mass-enhanced region only.
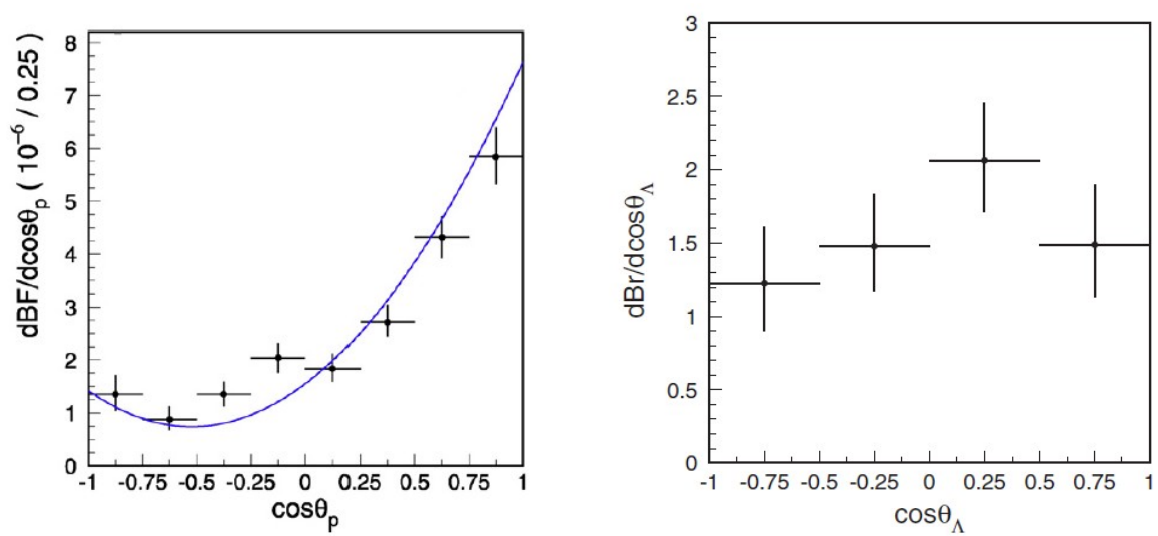

Figure 6: Differential branching fractions vs. $\cos \theta_{p}$ in the $p \bar{p}$ system for $B^{+} \rightarrow p \bar{p} K^{+}$decay (left) and $\cos \theta_{\Lambda}$ in the $\Lambda \bar{\Lambda}$ system for $B^{+} \rightarrow \Lambda \bar{\Lambda} K^{+}$decay (right), in the threshold-mass-enhanced region.

The polar angular distribution of the $\Lambda$ in the $\Lambda \bar{\Lambda}$ pair rest frame is also studied in the $B^{+} \rightarrow$ $\Lambda \bar{\Lambda} K^{+}$decay. In Figure 6, the signal yield is plot for each bin of $\cos \theta_{\bar{\Lambda}}$, where $\theta_{\bar{\Lambda}}$ is the angle between the $\bar{\Lambda}$ direction and the $K^{+}$direction in the $\Lambda \bar{\Lambda}$ pair rest frame. The distribution has no clear forward peak as seen in the $B^{+} \rightarrow p \bar{p} K^{+}$decay [17], which is also shown in the figure for comparison.

Comparing the $B \rightarrow p \bar{p} h$ and $B \rightarrow \Lambda \bar{\Lambda} h$ decays, the small value of $\mathscr{B}\left(B^{+} \rightarrow \Lambda \bar{\Lambda} \pi^{+}\right)$, the large value of $\mathscr{B}\left(B^{0} \rightarrow \Lambda \bar{\Lambda} K^{0}\right)$, and the lack of a peaking feature in the $\cos \theta_{\Lambda}$ distribution for $B^{+} \rightarrow \Lambda \bar{\Lambda} K^{+}$indicate that the underlying dynamics of the two types of decays are quite different.

\section{$2.3 B^{+} \rightarrow p \bar{\Lambda} \pi^{+} \pi^{-}$}

A hierarchy in branching fraction of charmed baryonic B decays has been experimentally established, namely, $\mathscr{B}\left(B^{0} \rightarrow p \bar{\Lambda}_{c}^{-} \pi^{+} \pi^{-}\right)>\mathscr{B}\left(B^{0} \rightarrow p \bar{\Lambda}_{c}^{-} \pi^{+}\right)>\mathscr{B}\left(B^{0} \rightarrow p \bar{\Lambda}_{c}^{-}\right)[5,9,21,22]$. Many three-body charmless baryonic B meson decays have been also studied in the recent years. Therefore it is interesting to investigate four-body charmless baryonic B decays and check the corresponding branching fraction hierarchy.

Belle collaboration has presented the first observation of the four-body charmless baryonic decay $B^{+} \rightarrow p \bar{\Lambda} \pi^{+} \pi^{-}$, based on a data sample of 657 million $B \bar{B}$ pairs [23]. They also observed 


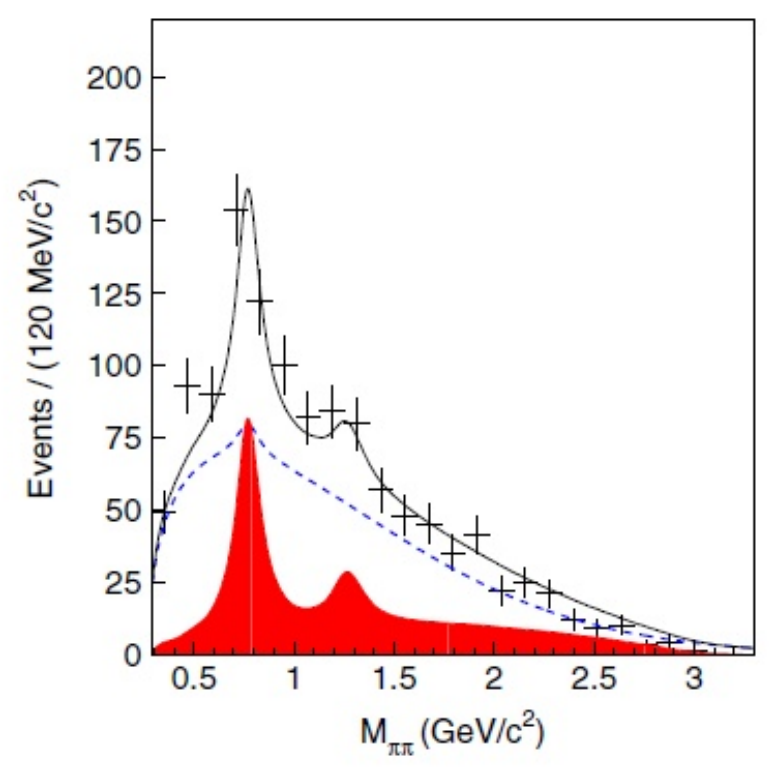

Figure 7: Distribution of $M_{\pi^{+} \pi^{-}}$within signal window. the solid curves represent the fit projection, which is the sum of signal and background (dashed curves) estimates. The shaded area represents the sum of signal components, i.e. $B^{+} \rightarrow p \bar{\Lambda} \pi^{+} \pi^{-}, B^{+} \rightarrow p \bar{\Lambda} \rho^{0}$, and $B^{+} \rightarrow p \bar{\Lambda} f_{2}(1270)$.

an intermediate three-body decays, $B^{+} \rightarrow p \bar{\Lambda} \rho^{0}$, and found a hint of a $B^{+} \rightarrow p \bar{\Lambda} f_{2}(1270)$ signal (Figure 7).

\begin{tabular}{llc}
\hline \hline Mode & $\mathscr{B}\left(10^{-6}\right)$ & significance \\
\hline$B^{+} \rightarrow p \bar{\Lambda} \pi^{+} \pi^{-}$ & $5.92_{-0.84}^{+0.88} \pm 0.69$ & 9.1 \\
$B^{+} \rightarrow p \bar{\Lambda} \rho^{0}$ & $4.78_{-0.64}^{+0.67} \pm 0.60$ & 9.5 \\
$B^{+} \rightarrow p \bar{\Lambda} f_{2}(1270)$ & $2.03_{-0.72}^{+0.77} \pm 0.27$ & 3.0 \\
\hline \hline
\end{tabular}

Table 3: The branching fractions and significances of the three decays obtained in the four-body charmless baryonic B decay study. The uncertainties are statistical and systematic ones.

The observed branching fraction for $B^{+} \rightarrow p \bar{\Lambda} \pi^{+} \pi^{-}$, excluding the two intermediate threebody states, is comparable to the corresponding three-body decay modes [15]. However, the central values may indicate that the hierarchy in branching fraction also applies in the charmless baryonic decay.

While studying the differential branching fraction as a function of the baryon-antibaryon mass, an enhancement near the baryon-antibaryon mass threshold is seen (Figure 8). The intriguing nearthreshold enhancement observed in three-body charmless baryonic B decays also holds in fourbody decays.

\section{Summary}

The large data sample of $B \bar{B}$ pairs gives a good opportunity to study the baryonic B meson 


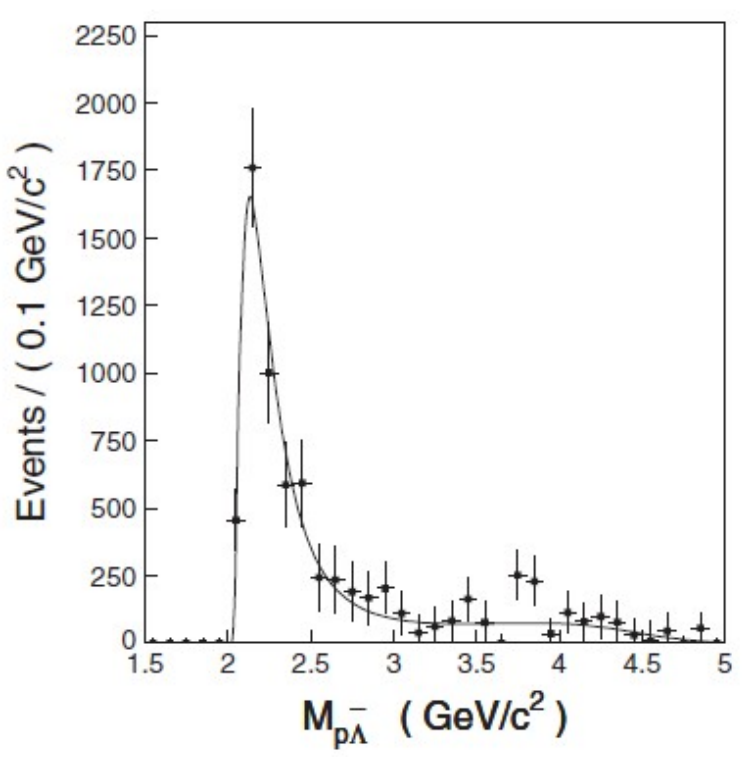

Figure 8: B signal yields as a function of the $p \bar{\Lambda}$ mass. The solid curve shows a fit with a threshold function.

decays. The profound baryonic B decays could be a searching ground for new physics and yetunknown exotic states.

In the recent studies done by BaBar and Belle collaborations, several new modes have been observed: $B^{-} \rightarrow \Sigma_{c}(2800)^{0} \bar{p}, B^{0} \rightarrow \Lambda \bar{\Lambda} K^{0}, B^{0} \rightarrow \Lambda \bar{\Lambda} K^{* 0}$, and the first observed charmless fourbody decay $B^{+} \rightarrow p \bar{\Lambda} \pi^{+} \pi^{-}$. A preliminary result of first evidence of semileptonic B decay is also presented.

The interesting hierarchy in branching fraction, $\mathscr{B}(4-b o d y)>\mathscr{B}(3-$ body $)>\mathscr{B}(2-$ body $)$, has been checked and holds in both charm and charmless baryonic modes. The enhancement near the invariant mass of baryon-antibaryon pair exists in all three- and four-body modes examined so far. In comparison between $B \rightarrow p \bar{p} h$ and $B \rightarrow \Lambda \bar{\Lambda} h$ decays shows that the underlying physics dynamics may be quite different.

\section{References}

[1] W. -S. Hou and A. Soni, Phys. Rev. Lett. 86 (2001) 4247.

[2] Throughout this report, inclusion of charge-conjugate modes is always implied unless otherwise stated.

[3] B. Aubert et al.(BaBar Collaboration), Phys. Rev. D 78 (2008) 112003.

[4] N. Gabyshev et al. (Belle Collaboration), Phys. Rev. Lett. 90 (2003) 121802.

[5] N. Gabyshev et al. (Belle Collaboration), Phys. Rev. Lett. 97 (2006) 242001.

[6] Throughout this report, the first uncertainty of any measurement is always the statistical one, and the second the systematic one, unless otherwise stated.

[7] B. Aubert et al. (BaBar Collaboration), Phys. Rev. D 80 (2009) 051105. 
[8] S. A. Dytman et al. (CLEO Collaboration), Phys. Rev. D 66 (2002) 091101.

[9] K. S. Park et al. (Belle collaboration) Phys. Rev. D 75 (2007) 011101.

[10] C. Amsler et al. (Particle Data Group) Phys. Lett. B 667 (2008) 1.

[11] M. E. Peskin, Nature(London) 452 (2008) 293.

[12] B. Aubert et al. (BaBar Collaboration), Phys. Rev. D 79 (2009) 112009.

[13] B. Aubert et al. (BaBar Collaboration), arXiv:0808.0011 (2008).

[14] W. M. Yao et al. (Partical Data Group), J. Phys. G 33 (2006) 1, and (2007) partial update for the 2008 edition.

[15] M. -Z. Wang et al. (Belle Collaboration), Phys. Rev. D 76 (2007) 052004.

[16] Y.-W. Chang et al. (Belle Collaboration), Phys. Rev. D. 79 (2009) 052006.

[17] J. -T. Wei et al. (Belle Collaboration), Phys. Lett. B 659 (2008) 80.

[18] M. Suzuki, J. Phys. G 34 (2007) 283.

[19] H. Y. Cheng and K. C. Yang, Phys. Rev. D 66 (2002) 014020.

[20] J. H. Chen et al. (Belle Collaboration), Phys. Rev. Lett. 100 (2008) 251801.

[21] X. Fu et al. (CLEO Collaboration), Phys. Rev. Lett. 79 (1997) 3125.

[22] N. Gabyshev et al. (Belle Collaboration), Phys. Rev. D. 66 (2002) 091102.

[23] P. Chen et al. (Belle Collaboration), Phys. Rev. D. 80 (2009) 111103. 